\title{
Caries dental y sus consecuencias clínicas relacionadas al impacto en la calidad de vida de preescolares de una escuela estatal
}

\begin{abstract}
Dental caries and its clinical consequences related to the impact on the quality of life of preschoolers of a state school
\end{abstract}

Liz Morales Miranda ${ }^{1, a}$, Walter Gómez Gonzáles 2,3,b,c;,d;;

\section{RESUMEN}

Objetivo: Determinar la presencia de caries dental y sus consecuencias clínicas relacionadas al impacto en la calidad de vida de preescolares de la institución educativa $\mathrm{N}^{\circ} 2051$ "El Progreso" en el 2018. Materiales y métodos: Estudio transversal y observacional. Se realizó una exploración clínica a 95 niños de 3-5 años, registrando la caries dental mediante los índices ceo-d y pufa. Se aplicó el ECOHIS dirigido a los responsables, para evaluar el impacto en la calidad de vida de los niños y sus familias. Las pruebas estadísticas utilizadas fueron t-Student, Kruskal-Wallis y U Mann-Whitney, con significancia 0,05; y Coeficiente de Spearman 0,01. Resultados: La frecuencia de caries dental 98,84\%, con índice ceo-d 9,58 3,45 correspondiente al nivel de severidad muy alto; y las consecuencias clínicas de caries dental no tratada $83,16 \%$, índice pufa $7,65 \pm 4,97$ y radio pufa $83,18 \%$. El ECOHIS mostró un promedio 23,41 111,82, siendo las dimensiones más afectadas limitación funcional y aspectos psicológicos, reflejando un impacto negativo en la calidad de vida relacionada a su salud bucal. Según el coeficiente de Spearman, la relación entre la presencia de caries dental $(\mathrm{r}=0,630)$ y sus consecuencias clínicas $(\mathrm{r}=0,649)$, con el impacto en la calidad de vida, muestran una correlación directa positiva moderada significativa $(\mathrm{p}=0,000$ ). Los índices del sexo masculino fueron ceo-d $24,46 \pm 12,11$ y pufa $28,88 \pm 7,03$; y del grupo de 5 años fueron ceo-d 29,71 $\pm 6,30$ y pufa 29,71 $\pm 6,30$. Conclusiones: La caries dental y sus consecuencias clínicas están relacionadas al impacto negativo en la calidad de vida a nivel bucal de los preescolares, interfiriendo en sus actividades diarias.

PALABRAS CLAVE: Caries dental, calidad de vida, salud bucal, preescolar.

Escuela Profesional de Estomatología, Universidad Alas Peruanas. Lima, Perú.

Escuela de Post Grado, Universidad Alas Peruanas. Lima,Perú.

Escuela de Post Grado, Universidad Andina del Cusco. Cusco,Perú.

Universidad Privada Telesup. Lima, Perú.

Dirección de Investigación, Universidad Privada Juan Pablo II. Lima, Perú.

Bachiller en Estomatología; b Profesor; c Doctor en Ciencias de la Salud, d Profesor Investigador. 


\section{SUMMARY}

Objectives: To determine the presence of dental caries and its clinical consequences related to the impact on the quality of life of preschool children of the educational institution $\mathrm{N}^{\circ} 2051$ "El Progreso" in 2018. Materials and methods: Cross-sectional and observational study. A clinical examination was performed on 95 children aged 3-5 years, recording dental caries by means of the ceo-d and pufa indexes. The ECOHIS was applied to those responsible, to assess the impact on the quality of life of children and their families. The statistical tests used were t-Student, Kruskal-Wallis and U Mann-Whitney, with significance 0.05; and Spearman coefficient 0.01. Results: The frequency of dental caries $98.84 \%$, with ceo-d index $9.58 \pm 3.45$ corresponding to the level of very high severity; and the clinical consequences of untreated dental caries $83.16 \%$, index pufa $7.65 \pm 4.97$ and radio frequency $83.18 \%$. The ECOHIS showed an average of $23.41 \pm 11.82$, the dimensions most affected being functional limitation and psychological aspects, reflecting a negative impact on the quality of life related to oral health. According to the Spearman coefficient, the relationship between the presence of dental caries $(\mathrm{r}=0.630)$ and its clinical consequences $(\mathrm{r}=0.649)$, with the impact on quality of life, show a positive direct correlation moderate significant $(\mathrm{p}=0.000)$. The male sex indexes were ceo-d $24.46 \pm 12.11$ and pufa $28.88 \pm 7.03$; and of the group of 5 years were ceo-d $29.71 \pm 6.30$ and pufa $29.71 \pm 6.30$. Conclusions: Dental caries and its clinical consequences are related to the negative impact on the quality of life at the oral level of preschoolers, interfering with their daily activities.

\section{KEY WORDS: Dental caries, quality of life, oral health, preschool.}

\section{INTRODUCCIÓN}

La caries dental es una patología de etiología multifactorial, transmisible de origen infeccioso que afecta a las piezas dentarias, produciendo la destrucción de forma progresiva de los tejidos duros (1). Estudios reportados a nivel mundial sobre su prevalencia, tales como la Organización Mundial de la Salud (OMS) en el 2004, mostró un 60 a $90 \%$ en escolares y casi el $100 \%$ en adultos (2), y la Federation Dental International (FDI) en el 2010, encontró un 44\%, afectando casi la mitad de la población (3). En el Perú, el Ministerio de Salud (MINSA) en el 2005, reportó una prevalencia de $90 \%$ en escolares, donde el promedio del índice ceo-d fue de 5,84 (4). Estas cifras son alarmantes, evidenciando que no discrimina edad, sexo, ni condición socioeconómica.

Sin un abordaje terapéutico oportuno de la remoción de caries, ocurrirá una inflamación pulpar (pulpitis reversible), progresando hacia una pulpitis irreversible, y acabando con la vitalidad de la pieza dental (necrosis pulpar), trayendo consigo la destrucción y pérdida del órgano dental (5). Esto puede generar alteraciones a nivel funcional, estético y psicosocial donde las consecuencias en el paciente pediátrico pueden ser la disminución de la autoestima, cambios en el desenvolvimiento y comportamiento a nivel social pudien- do ocasionar con el tiempo, aislamiento y ausentismo escolar. En el aspecto funcional se podrá observar una deficiencia masticatoria, originando una alteración en la nutrición y alimentación del niño, como también dificultad en el habla (6).

Las lesiones cariosas no tratadas repercuten no sólo en el desarrollo y crecimiento del niño, sino que pueden llegar a ocasionar la muerte del infante, como fue reportado en el 2007, en el estado de Maryland en los Estados Unidos, la muerte de un niño de 12 años de edad, debido a caries dentales no tratadas que dió como resultado una infección cerebral (7).

Estas consecuencias repercuten en la calidad de vida, cuya definición según la OMS, es la percepción individual de cada persona de su estado actual de vida, dentro del ámbito social y cultural, además de los valores en el que habita, asociado con sus propósitos, aspiraciones y aptitudes (8).

En los últimos años, han aparecido estudios sobre la calidad de vida donde ha sido vinculada con la salud, específicamente en el ámbito odontológico, dando origen al término "Calidad de vida relacionada a la salud bucal" (CVRSB), definido como la percepción que tiene cada persona sobre el grado de disfrute de su cavidad oral, involucrando tanto a los tejidos duros 
como blandos, a lo largo de sus labores diarias, considerando los tratamientos odontológicos pasados y actuales, sus intereses, complacencia con la atención, historia del dolor dental y el malestar (9). Locker en 1988 creó un modelo de estudio teórico de tres niveles, con el objetivo de dar a conocer la relación de la salud bucal con el impacto en las actividades diarias: Primer nivel, indica la condición oral, incluyendo deterioros bucodentales; segundo nivel, o impactos intermedios, son los impactos negativos iniciales a causa de la condición de salud oral como el dolor, limitación funcional e insatisfacción con su apariencia; tercer nivel, o impactos finales, que representan aquellos impactos con la capacidad de realizar las actividades diarias, donde se requiere un desempeño físico adecuado, psicológica y social (10).

La importancia del estudio, radica en la poca evidencia de reportes sobre las consecuencias clínicas de la caries dental no tratada medidas con el índice pufa, y un menor número de investigaciones que la relacionen con la calidad de vida en los preescolares, el cual es un grupo susceptible y de alto riesgo a enfermedades bucodentarias; además de la poca importancia que los responsables del cuidado del infante muestran en la dentición decidua sobre las repercusiones que conlleva este problema en los diferentes ámbitos de su vida.

El objetivo del presente trabajo fue determinar la presencia de caries dental y sus consecuencias clínicas relacionadas al impacto en la calidad de vida de preescolares de la institución educativa $\mathrm{N}^{\circ} 2051$ "El Progreso" ubicado en el distrito de Carabayllo, Lima - Perú en el año 2018.

\section{MATERIAL Y MÉTODOS}

Estudio de tipo observacional, descriptivo y de corte transversal; con un universo de 173 niños de 3-5 años de la I.E. N N $^{\circ} 2051$ "El Progreso" ubicado en el distrito de Carabayllo, y una muestra de 95 niños, la cual se determinó utilizando una fórmula para poblaciones finitas, a través de un cálculo para estimar una media, seleccionados mediante un muestreo probabilístico estratificado simple.

Los criterios de inclusión fueron infantes matriculados de ambos géneros entre 3 a 5 años, cooperadores y padres o apoderados que aceptaron mediante su firma en el consentimiento informado su participación.
Además, los criterios de exclusión fueron niños con compromiso sistémico o tratamiento farmacológico que puedan provocar la alteración de la cavidad oral, y que sus padres o apoderados no presenten ninguna discapacidad mental auditiva, y cognitiva.

Para el proceso de recolección de datos, se reunió a los padres de familia por aulas, previa convocatoria realizada por la dirección, a través de una invitación anexada a la agenda del menor. Se les explicó los objetivos y procedimientos del estudio y se les alcanzó el consentimiento informado, haciendo mención que la participación es voluntaria. Los padres de los menores que fueron seleccionados aleatoriamente, se les solicitó que firmen este documento. Posteriormente, se procedió a realizarles la encuesta multidimensional denominada ECOHIS (Early Childhood Oral Health Scale) a través de una entrevista estructurada, para medir el impacto de los problemas orales en relación a la calidad de vida en los preescolares y su entorno.

Este cuestionario fue traducido y validado al idioma español por Bordoni et al.,(11), para la aplicación en distintos lugares de América Latina. Además, se le realizó la adaptación transcultural y validación de la versión peruana al ECOHIS, siendo utilizada en distintas investigaciones en el Perú. Presenta una confiabilidad de acuerdo a su consistencia interna, la cual fue medida a través del Coeficiente del Alpha de Cronbach de 0.925 para el dominio del impacto sobre el niño, y 0.882 para el impacto del dominio Familia, y 0.948 para la valoración en general del ECOHIS. Se explicaron las 13 preguntas, distribuidas en 2 dominios: Impacto sobre el niño con 4 subdivisiones (síntomas orales, limitaciones funcionales, aspectos psicológicos y autoimagen/ interacción social) e impacto familiar con 2 subdivisiones (angustia de los padres y función familiar). Cada ítem tenía las alternativas: Nunca, Casi nunca, Ocasionalmente, A menudo y Muy a menudo, dando un valor de 0 a 4 puntos, respectivamente. La sumatoria de las puntuaciones de cada una de las preguntas se encontraban en un rango de 0-52 puntos, de modo que a mayor puntaje, mayor sería el impacto negativo en la calidad de vida del niño. Las encuestas que tuvieron marcadas 2 veces o más la alternativa de No sabe, fueron anuladas $(12,13)$. 
La ficha clínica contenía los datos de filiación del niño, el examen dental y los índices ceo-d y pufa. Previo a la realización del examen clínico, se llevó a cabo una charla informativa y de estimulación a los menores, con el fin de afianzar la confianza y su familiarización con los instrumentos a utilizar en la evaluación diagnóstica.

Se realizó la inspección clínica bajo los criterios de la OMS (14), en las piezas dentales deciduas para detectar la presencia de caries dental y sus consecuencias clínicas por medio del método visual en un aula ambientada para la atención odontopediátrica. Se inició con el lavado de manos del examinador con jabón líquido, y la colocación de guantes de examen, mascarilla y gorro descartable. Se ubicó el examinador sentado frente al niño. Se procedió a la limpieza de las piezas dentarias con gasa y agua, antes de la evaluación clínica realizada con frontoluz y equipo de diagnóstico conformado por espejo bucal, pinza para algodón y baja lengua.

El índice ceo-d fue categorizado en rangos según el nivel de severidad establecido por la OMS: Muy Bajo $(0-1,2)$, Bajo $(1,3-2,6)$, Moderado $(2,7-4,4)$, Alto $(4,5-6,5)$ y Muy Alto $(>6,5)$. Las lesiones incipientes (mancha blanca), no son consideradas (14).

El índice pufa determina la gravedad y extensión de las condiciones orales en dentición decidua, para el registro cuantitativo de las consecuencias clínicas de la caries no tratada, teniendo como indicadores: afección pulpar (p), cuando la exposición pulpar es visible; ulceración (u), presencia de traumatismos en tejidos blandos por fragmentos radiculares; fistula (f), cuando la pus que se libera del tracto sinusal está relacionada con un diente con afección pulpar; y absceso (a), acumulo de pus que está relacionada con un diente con afección pulpar. La puntuación de cada niño, se calculó mediante la sumatoria de las piezas afectadas, encontrándose en un rango de 0 a 20 puntos. La prevalencia se presentó en porcentaje. La experiencia de pufa poblacional fue calculada como un promedio. La caries no tratada-radio pufa, se halló por la fórmula [pufa/dientes cariados] x 100, indicando el porcentaje de dientes con caries sin abordaje oportuno que desarrollaron infecciones odontogénicas. Este índice provee información sobre las consecuencias clínicas tales como la afección pulpar y abscesos dentales, siendo un complemento del índice ceo-d, permitien- do realizar un análisis completo de la caries dental, ya que este último solo se enfoca en cuantificar las experiencias de lesiones cariosas y tratamientos previos $(15,16)$.

En cuanto a las consideraciones éticas, la investigación fue revisada y aprobada por el Comité Institucional de Ética de la Universidad Alas Peruanas. Además, se obtuvo el consentimiento informado de los padres y se mantuvieron los datos recaudados en reserva.

El procesamiento de datos se realizó mediante el programa estadístico SPSS versión 25. Se empleó las pruebas estadísticas paramétricas (t de Student) y no paramétricas ( $\mathrm{H}$ de Kruskal-Wallis y la U de Mann-Whitney), con previo análisis de normalidad (Kolmogórov-Smirnov). Para establecer la fuerza de correlación entre las dos variables, se utilizó el Coeficiente de Spearman con un nivel de significancia de 0,01 , indicando la calidad y veracidad de los datos.

\section{RESULTADOS}

Se evaluaron 95 niños con edades que varían de 3 a 5 años $(4,05 \pm 0,82)$, donde la mayor proporción se concentró en el grupo de 5 años $(35,79 \%)$ y en niños varones $(52,63 \%)$ (tabla 1$)$.

En el $12,63 \%$ de los preescolares, se observa un índice ceo-d de 8 , seguido de un $11,58 \%$ con un ceo-d de 6. El promedio total del índice ceo-d fue 9,58 \pm 3,45 ; considerado como un nivel de severidad de caries dental muy alto, comprendido por 72 preescolares $(75,79 \%)$. E1 98,84\% de los prescolares presentan caries dental (tabla 2).

El 11,6\% de los niños presentaron un índice de pufa de 6 puntos, seguido de $8,4 \%$ con índice pufa de 13 , 8 y 7 puntos. La prevalencia de pufa fue $83,16 \%$ y el promedio fue 7,65 $\pm .4,97$. El componente con mayor valor fue " $p$ " que mostró el mayor valor $(6,45 \pm 4,60)$ y un puntaje de 15 . La caries no tratada-radio pufa fue de $83,18 \%$, indicando que el componente "c" del índice ceo-d ha progresado a una infección odontogénica (tabla 3).

No se encontró diferencia significativa según sexo. Además, en el grupo de 5 años, el índice pufa tuvo una prevalencia del 100\%, con una puntuación máxi- 
ma de 16 , y promedio más alto de $13,15 \pm 1,73$ en comparación con las edades de 3 años $(2.41 \pm 2,75)$ y 4 años $(6,56 \pm 2,21)$. Hay diferencia estadísticamente significativa $(p<0,001)$ entre los valores promedios del índice pufa y la edad del preescolar (tabla 4).

La puntuación del ECOHIS total máxima fue 42 y la mínima 0 , con un promedio de $23,41 \pm 11,82$; evidenciando un impacto negativo en la calidad de vida de los preescolares. En la sección de impacto en el niño, el promedio fue $16,89 \pm 8,60$, donde la dimensión limitación funcional obtuvo el promedio más alto $7,34 \pm 3,96$; seguido por alteraciones psicológicas con $3,42 \pm 1,86$. Mientras que, en el impacto en la familia, mostró un promedio de $6,49 \pm 4,03$, donde la dimensión angustia de los padres fue el más alto con $3,58 \pm 2,14$ (tabla 5).

Al relacionar el ECOHIS con la presencia caries dental, el grupo con muy alta severidad presenta el mayor promedio $28,03 \pm 7,77$; en comparación con el grupo de baja severidad con un menor promedio de $0,40 \pm$
0,89; con coeficiente de Spearman $(\mathrm{r}=0,630)$. Mientras que, las consecuencias clínicas de la caries dental no tratada muestran un alto promedio $27,96 \pm 6,57$ en relación con la calidad de vida; con coeficiente de Spearman $(\mathrm{r}=0,649)$. Mostrando ambas relaciones significativas $(\mathrm{p}<0,001)$ (tabla 6$)$.

Según el sexo del preescolar, hubo un predominio en el sexo masculino para la caries dental, y sus consecuencias clínicas de la caries dental no tratada; con un coeficiente de Spearman de 0,713 y 0,862 , respectivamente, siendo ambas significativas $(\mathrm{p}<0,001)$ (tabla 7)

Según la edad del preescolar, la relación entre las variables, mostró un predominio en el grupo de 5 años con un mayor promedio de $29,71 \pm 6,30$ para la caries dental, y 29,71 $\pm 6,30$ para las consecuencias clínicas de la caries dental no tratada; con un coeficiente de Spearman de 0,596 y 0,804 , respectivamente, siendo ambas significativas $(\mathrm{p}<0,001)$ (tabla 8$)$.

Tabla 1. Características del preescolar y del responsable de su cuidado en la institución educativa $\mathrm{N}^{\circ}$ " 2051 ” -2018

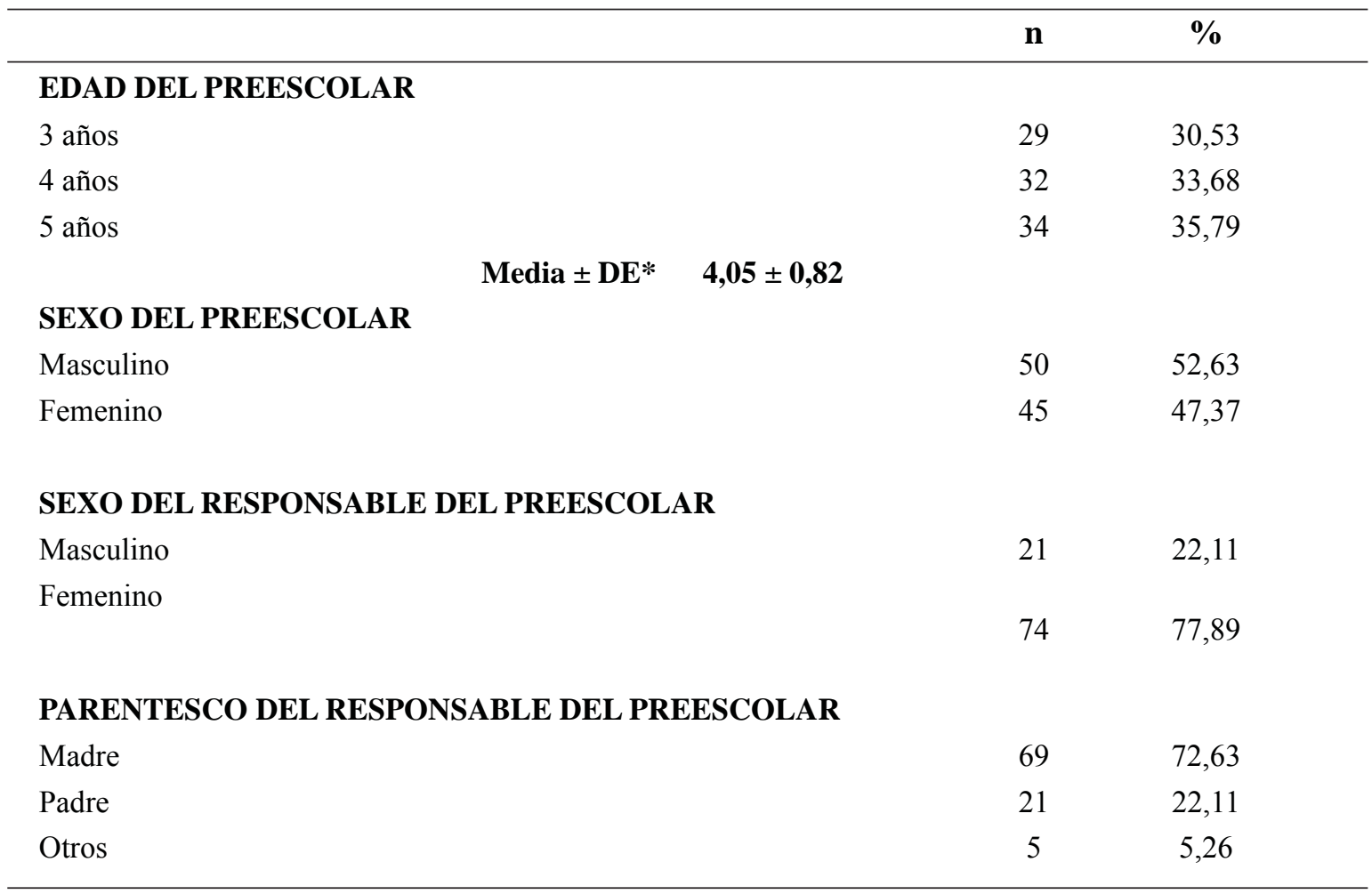

Fuente propia del investigador

* $\mathrm{DE}=$ Desviación estándar 
Tabla 2. Frecuencia de la presencia de caries dental de preescolares de la institución educativa N² 2051 "El Progreso" - 2018

\begin{tabular}{|c|c|c|}
\hline ceo-d & $\mathbf{n}$ & $\%$ \\
\hline 0 & 5 & 5,25 \\
\hline 1 & 1 & 1,05 \\
\hline 3 & 3 & 3,16 \\
\hline 4 & 2 & 2,11 \\
\hline 5 & 2 & 2,11 \\
\hline 6 & 11 & 11,58 \\
\hline 7 & 5 & 5,26 \\
\hline 8 & 12 & 12,63 \\
\hline 9 & 8 & 8,42 \\
\hline 10 & 4 & 4,21 \\
\hline 11 & 6 & 6,32 \\
\hline 12 & 4 & 4,21 \\
\hline 13 & 10 & 10,53 \\
\hline 14 & 7 & 7,37 \\
\hline 15 & 9 & 9,47 \\
\hline 16 & 6 & 6,32 \\
\hline Índice ceo-d & Media \pm DE & $9,58 \pm 3,45$ \\
\hline Nivel de Severidad & $\mathbf{n}$ & $\%$ \\
\hline Muy bajo & 5 & 5,26 \\
\hline Bajo & 0 & 0,00 \\
\hline Moderado & 5 & 5,26 \\
\hline Alto & 13 & 13,69 \\
\hline Muy alto & 72 & 75,79 \\
\hline Experiencia de caries & $\mathbf{n}$ & $\%$ \\
\hline No presenta & 3 & 3,16 \\
\hline
\end{tabular}


Tabla 3. Frecuencia de las consecuencias clínicas de la caries dental de preescolares de la institución educativa N² 2051 "El Progreso" - 2018

\begin{tabular}{ccccccc}
\hline & $\mathbf{n}$ & $\mathbf{\%}$ & media & DE & Min. & Max. \\
\hline $\mathbf{p}$ & 79 & 83,16 & 6,45 & 4,60 & 0 & 15 \\
$\mathbf{f}$ & 24 & 25,26 & 0,35 & 0,66 & 0 & 3 \\
$\mathbf{a}$ & 11 & 11,58 & 0,15 & 0,44 & 0 & 2 \\
Índice pufa & 51 & 53,68 & 0,71 & 0,76 & 0 & 3 \\
Sin pufa & 79 & $83,16 \%$ & 7,65 & 4,97 & 0 & 16 \\
Caries no tratada - radio pufa & 16 & $16,84 \%$ & 0,00 & 0,00 & - & - \\
\hline
\end{tabular}

*DE= Desviación estándar

Tabla 4. Frecuencia de las consecuencias clínicas de la caries dental de preescolares de la institución educativa N² 2051 "E1 Progreso" - 2018, según sexo y edad.

\begin{tabular}{|c|c|c|c|c|c|c|c|c|}
\hline & & $\mathbf{n}$ & media & DE & Mín. & Máx. & $\begin{array}{c}\text { Con experiencia } \\
\text { pufa } n(\%)\end{array}$ & Valor $p$ \\
\hline \multirow[t]{4}{*}{ Sexo } & Masculino & 50 & 7,84 & 4,93 & 0 & 16 & $42(84,00 \%)$ & \\
\hline & o & & & & & & & $0.776^{\mathrm{a}}$ \\
\hline & Femenino & 45 & 7,44 & 5,07 & 0 & 16 & $37(82,22 \%)$ & \\
\hline & 3 años & 29 & 2.41 & 2,75 & 0 & 8 & $15(51,72 \%)$ & \\
\hline \multirow[t]{2}{*}{ Edad } & 4 años & 32 & 6,56 & 2,21 & 0 & 9 & $30(93,75 \%)$ & $<\mathbf{0 , 0 0 1} 1^{\mathrm{b}}$ \\
\hline & 5 años & 34 & 13,15 & 1,73 & 9 & 16 & $34(100,00 \%)$ & \\
\hline
\end{tabular}

$\mathrm{DE}=$ Desviación estándar

a Prueba t para muestras independientes; $\mathrm{p}<0,05$.

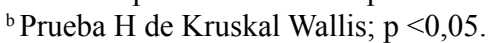


Tabla 5. Impacto en la calidad de vida de preescolares de la institución educativa N 2051 "El Progreso" - 2018

\begin{tabular}{|c|c|c|c|c|c|}
\hline \multirow{2}{*}{ Dimensiones del ECOHIS } & \multirow{2}{*}{$\begin{array}{l}\text { Número de } \\
\text { preguntas }\end{array}$} & \multicolumn{3}{|c|}{ Impacto en la calidad de vida } & \multirow{2}{*}{$\begin{array}{l}\text { Rango posible del } \\
\text { ECOHIS }\end{array}$} \\
\hline & & Media & DE & Rango obtenido & \\
\hline Puntuación total del ECOHIS & 13 & 23,41 & 11,82 & $0-42$ & $0-52$ \\
\hline \multicolumn{6}{|l|}{ Dimensiones } \\
\hline Impacto en el niño & 9 & 16,89 & 8,60 & $0-28$ & $0-36$ \\
\hline Síntomas Orales & 1 & 2,77 & 1,34 & $0-4$ & $0-4$ \\
\hline Limitaciones funcionales & 4 & 7,34 & 3,96 & $0-13$ & $0-16$ \\
\hline Alteraciones psicológicas & 2 & 3,42 & 1,86 & $0-6$ & $0-8$ \\
\hline Autoimagen/interacción social & 2 & 3,39 & 2,08 & $0-8$ & $0-8$ \\
\hline Impacto en la familia & 4 & 6,49 & 4,03 & $0-14$ & $0-16$ \\
\hline Angustia de los padres & 2 & 3,58 & 2,14 & $0-8$ & $0-8$ \\
\hline Función familiar & 2 & 2,92 & 2,15 & $0-8$ & $0-8$ \\
\hline
\end{tabular}

$\mathrm{DE}=$ Desviación estándar

Tabla 6. Presencia de caries dental y sus consecuencias clínicas relacionadas al impacto en la calidad de vida de preescolares de la institución educativa N²051 “El Progreso" - 2018.

\begin{tabular}{|c|c|c|c|c|c|c|}
\hline \multirow{2}{*}{$\begin{array}{l}\text { Presencia de Caries Dental y sus } \\
\text { consecuencias clínicas }\end{array}$} & \multicolumn{6}{|c|}{ Impacto en la calidad de vida } \\
\hline & $\mathbf{n}$ & $\%$ & Media & DE & r (Spearman) & Valor $\mathbf{p}$ \\
\hline \multicolumn{7}{|l|}{ Presencia de Caries Dental } \\
\hline Muy Baja severidad & 5 & 5,26 & 0,40 & 0,89 & \multirow{4}{*}{$0,630 * *$} & \multirow{4}{*}{$<\mathbf{0 , 0 0 1}{ }^{\mathrm{a}}$} \\
\hline Moderada severidad & 5 & 5,26 & 1,60 & 2,61 & & \\
\hline Alta severidad & 13 & 13,69 & 15,08 & 10,65 & & \\
\hline Muy Alta severidad & 72 & 75,79 & 28,03 & 7,77 & & \\
\hline \multicolumn{7}{|c|}{$\begin{array}{l}\text { Consecuencias clínicas de Caries Dental } \\
\text { no tratada }\end{array}$} \\
\hline Ausencia & 16 & 16,84 & 0,94 & 1,91 & \multirow{2}{*}{$0,649 * *$} & \multirow[t]{2}{*}{$<0,001^{b}$} \\
\hline Presencia & 79 & 83,16 & 25.75 & 7,35 & & \\
\hline
\end{tabular}

DE: Desviación estándar

**Coeficiente Spearman: La correlación es significativa en el nivel 0,01 (bilateral)

${ }^{\text {a }}$ Prueba H de Kruskal Wallis; $\mathrm{p}<0,05$.

${ }^{\mathrm{b}}$ Prueba U de Mann Whitney; $\mathrm{p}<0,05$. 
Tabla 7. Presencia de caries dental y sus consecuencias clínicas relacionadas al impacto en la calidad de vida de preescolares de la institución educativa N²051 "El Progreso" - 2018, según sexo

\begin{tabular}{|c|c|c|c|c|c|c|}
\hline & \multirow{2}{*}{$\begin{array}{l}\text { Presencia de caries Dental y sus } \\
\text { consecuencias clínicas }\end{array}$} & \multicolumn{5}{|c|}{ Impacto en la calidad de vida } \\
\hline & & $\mathbf{n}$ & $\%$ & Media & DE & Valor $p$ \\
\hline \multirow{9}{*}{ Masculino } & Caries Dental & & & & & \multirow{9}{*}{$<0,001^{*}$} \\
\hline & Muy Baja severidad & 2 & 4,00 & 1,00 & 1,41 & \\
\hline & Moderada severidad & 3 & 6,00 & 2,67 & 3,06 & \\
\hline & Alta severidad & 9 & 18,00 & 15,11 & 10,97 & \\
\hline & Muy Alta severidad & 36 & 80,00 & 29,92 & 7,21 & \\
\hline & Total & 50 & 100 & 24,46 & 12,11 & \\
\hline & $\begin{array}{l}\text { Consecuencias clínicas de CD no } \\
\text { tratada }\end{array}$ & & & & & \\
\hline & Ausencia & 8 & 16,00 & 1,25 & 2,12 & \\
\hline & Presencia & 42 & 84,00 & 28,88 & 7,03 & \\
\hline \multirow{9}{*}{ Femenino } & Caries Dental & & & & & \multirow{9}{*}{$<0,001 *$} \\
\hline & Muy Baja severidad & 3 & 6,00 & 0,00 & 0,00 & \\
\hline & Moderada severidad & 2 & 4,00 & 0,00 & 0,00 & \\
\hline & Alta severidad & 4 & 8,00 & 15,00 & 11,52 & \\
\hline & Muy Alta severidad & 36 & 72,00 & 26,14 & 7,95 & \\
\hline & Total & 45 & 100 & 22,24 & 11,52 & \\
\hline & $\begin{array}{l}\text { Consecuencias clínicas de CD no } \\
\text { tratada }\end{array}$ & & & & & \\
\hline & Ausencia & 8 & 17,78 & 0,63 & 1,77 & \\
\hline & Presencia & 37 & 82,22 & 26,92 & 5,94 & \\
\hline
\end{tabular}

DE: Desviación estándar

CD: Caries Dental

**Coeficiente Spearman: La correlación es significativa en el nivel 0,01

Según el sexo, el Coeficiente de Spearman entre la CD y el impacto en la calidad de vida fue $r=0,713$; y las consecuencias clínicas de CD no tratada y el impacto en la calidad de vida fue $r=0,862$.

$*$ En ambos sexos, el valor $p$ obtenido fue $<0,001$, siendo significativas. 
Tabla 8. Presencia de caries dental y sus consecuencias clínicas relacionadas al impacto en la calidad de vida de preescolares de la institución educativa N 2051 "El Progreso" - 2018, según edad.

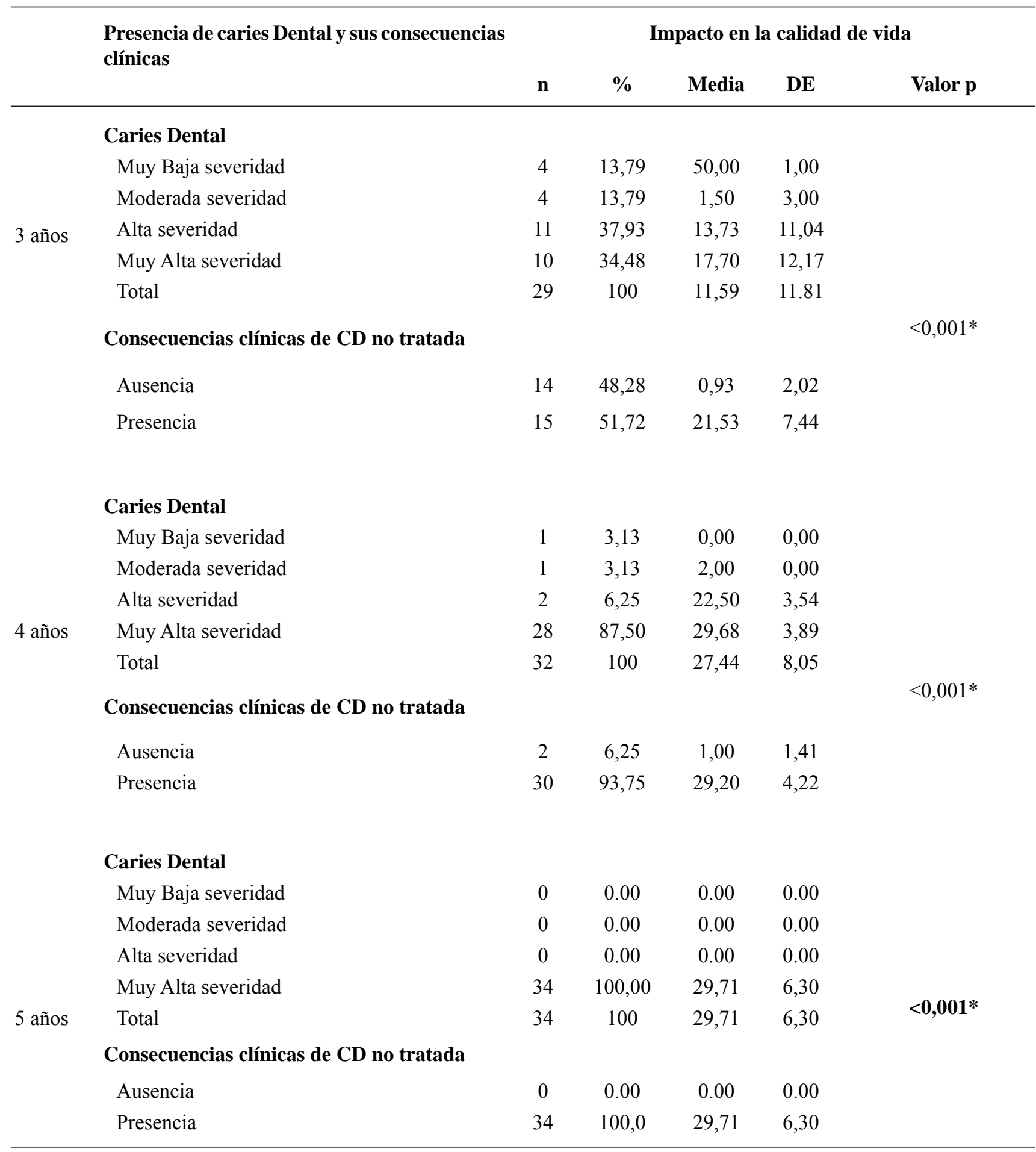

CD: Caries Dental

DE: Desviación estándar

**Coeficiente Spearman: La correlación es significativa en el nivel 0,01

Según la edad, el Coeficiente de Spearman entre la CD y el impacto en la calidad de vida fue $r=0,596$; y las consecuencias clínicas de CD no tratada y el impacto en la calidad de vida fue $\mathrm{r}=0,804$.

*En todas las edades, el valor $\mathrm{p}$ obtenido fue $<0,001$, siendo significativas. 


\section{DISCUSIÓN}

En el estudio se evaluó uno de los problemas bucodentales de mayor prevalencia en el mundo, como es la caries dental, encontrándose una alta frecuencia de casi el $100 \%$ en la población preescolar de 3 a 5 años de edad. Así lo reportan estudios realizados en niños de 2 a 5 años, como Bansal et al., $85 \%$ en la India (17), y Zambrano et al., 79,5\% en Venezuela (18). Así mismo, en niños de 3 a 5 años, reportaron Alosaimi et al., 85,5\% en Arabia Saudita (19); Quispe y Quintana 74\% (20), y López y García, 76,47\% en Perú (21). Además, Bagińska et al., encontraron un $85,9 \%$ en niños de 5 años en Polonia (22). Por lo contrario, otros autores no coinciden con las cifras reportadas, como Tello et al., 33,1\% en niños de 3 y 4 años en Brasil (23); y Duarte 20,3\% en niños de 3 a 5 años en Portugal (6). Estas diferencias podrían estar relacionadas a educación oral, hábitos de higiene, alimentación e interés parental.

El promedio del índice ceo-d fue de 9,58 $\pm 3,45$, indicando un nivel de severidad muy alto; coincidiendo con Bansal et al., con 7,13 $\pm 5,24$ en niños de 2 a 5 años en la India (17). Sin embargo, otros estudios obtuvieron niveles inferiores a los obtenidos. Alosaimi B et al., con nivel alto de 5,54 $\pm 3,49$ en Arabia Saudita (19); Patil et al., con nivel bajo de 2,57 $\pm 0,56$ en India (24); y Duarte I, con nivel muy bajo de 0,71 en Portugal (6) en niños de 3 a 5 años. Así mismo, Bagińska et al., obtuvieron 5,56 $\pm 4,45$ con nivel alto en niños de 5 años en Polonia (22); Tello et al., hallaron 3,07 con nivel moderado, en niños de 3 y 4 años en Brasil (23). Las diferencias encontradas podrían responder a factores socioeconómicos y falta de gestión pública de los gobiernos.

Al no dar tratamiento a la caries dental, se dan las primeras manifestaciones de destrucción o afección a nivel periapical de las piezas dentales deciduas, que se denominan consecuencias clínicas. Los preescolares evaluados en el estudio obtuvieron una alta frecuencia del índice pufa de $83,16 \%$. Esta cifra coincide con Bansal et al., con $61 \%$ en niños de 2 a 5 años en la India (17); Bagińska et al., 43,4\% en niños de 5 años en Polonia (22), a razón de la carencia de promoción y prevención de salud oral masiva, nutrición y desinterés de los responsables del infante. Por lo contrario, el seguimiento a la salud de la comunidad, podría generar resultados un poco más alentadores para el bienestar del infante como lo reportado por Patil et al., 24\% en India (24), y Alosaimi et al., 22,6\% en Arabia Saudita (19), en niños de 3 a 5 años.

Se identificó un alto promedio del índice pufa de 7,65 $\pm .4,97$, concentrándose en su gran mayoría en el componente "p" con $6,45 \pm 4,60$. Estas cifras promedio/ componente " $p$ ", difieren de: Alosaimi et al., 0,67 \pm 1,74/0,62 $\pm 1,74$ en Arabia Saudita (19); y Patil et al., $1,56 \pm 0,45 / 1,56 \pm 0,40$ en India (24), en niños de 3 a 5 años; Bagińska et al., 2,20 \pm . 3,43/2,13 $\pm 3,35$ en niños de 5 años en Polonia (22); Bansal et al., 2,18 \pm $2,82 /$ con prevalencia del componente "p" de 56,6\% en niños de 2 a 5 años en la India (17). Estas cifras podrían deberse al número de individuos estudiados, intervalos de edad situación socioeconómico y geográfico donde se realizó el estudio; y reflejarían una posible combinación entre factores como las deficiencias de un sistema de prevención, la falta de conocimiento parental y la limitación económica, teniendo repercusiones a nivel oral del menor.

Hay diversas alteraciones que afectan a las actividades cotidianas del menor, siendo muchas veces pasadas por alto por los responsables de su cuidado; para ello, existe un instrumento que permite evaluar el impacto en la calidad de vida de los niños en edad preescolar en el ámbito de la salud bucal llamada ECOHIS. En el estudio, se encontró un impacto negativo en la calidad de vida del infante; coincidiendo con estudios en niños de 3 a 5 años, como Mamani y Padilla, 24,9 $\pm 8,3$ (25); Quispe y Quintana, 11,67 $\pm 10,15$ (20); y López y García, 15,50 \pm 10,24 (21); y López et al., $17,02 \pm 10,16$ (12), en Perú. De manera contraria, en la literatura se evidencian promedios más bajos; los cuales pueden ser causados por la aplicación de un sistema de salud integral poco más eficientes según la localidad, el desinterés por parte de los padres, y su bajo nivel educativo y sociocultural; como se da en estudios reportados en niños de 2 a 5 años, por Kramer et al., 0,9 $\pm 3,09$ en Brasil (26); Díaz et al., $5,05 \pm 0,45$ en Colombia (27); y Ruano, 15,74 \pm 7,33 en Ecuador (28).

La pérdida de funcionalidad a nivel oral en el infante, genera problemas que afectan distintos ámbitos en su calidad de vida, las cuales son reflejadas en las dimensiones con más altos promedios en la sección de impacto en el niño del ECOHIS, como limitación funcional/alteraciones psicológicas con 7,34 $\pm 3,96$ 
y $3,42 \pm 1,86$, respectivamente; coincidiendo con lo reportado por Quispe y Quintana, 3,09 $\pm 3,43 / 1,93 \pm$ 1,89 (20); López y García C, 4,75 $\pm 3,44 / 2,31 \pm 1,79$ (21); y López et al., 5,22 $\pm 3,42 / 2,59 \pm 1,78$ (12), en niños de 3 a 5 años en Perú. Otros estudios discrepan con las puntuaciones de las investigaciones mencionadas; las cuales son relacionadas a ciertas alteraciones ocasionadas por el dolor, como el impedimento de realizar la masticación, el beber, hasta poder llegar a una ausencia escolar, además de trastornos en el sueño y frustración; hallando a la limitación funcional/síntomas orales los más frecuentes, siendo aplicados en menores de 2 a 5 años, como Kramer et al., con $0,30 \pm 1,16 / 0,17 \pm 0,53$ en Brasil (26); y Díaz et al., $1,10 \pm 0,13 / 0,5 \pm 0,05$ en Colombia (27).

Aun siendo el malestar propio del preescolar en su calidad de vida, este influye en su familia, de manera que, la experiencia de dolor que sufren los niños, conlleva al estrés y dificultades en varios aspectos de la vida cotidiana de los padres, lo cual, es reflejado en la dimensión de mayor promedio a través de la encuesta ECOHIS, donde encontramos que, en la sección del impacto en la familia, la dimensión angustia de los padres tuvo un promedio de $3,58 \pm 2,14$, donde el dolor en el niño, conlleva al estrés y dificultad en la vida de los padres. Este valor tiene similitud en trabajos en niños de 3 a 5 años, por Quispe y Quintana, 2,59 \pm 1,96 (20); López y García, 2,71 (21); López et al.,

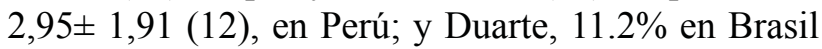
(6). En estudios a menores de 2 a 5 años: Zambrano et al., $15,7 \%$ en Venezuela (18); Kramer et al., 0,18 $\pm 0,86$ en 2 a 5 años en Brasil (26); y Diaz, 1,28 en Colombia (27). Estos promedios indican un menor o mayor impacto negativo en la familia.

En la relación del ECOHIS con la caries dental, se muestra un impacto negativo en la calidad de vida del menor, evidenciándose un nivel de severidad muy alto $28,03 \pm 7,77$, con valor de Spearman $\mathrm{r}=0,630$, siendo estadísticamente significativo $(\mathrm{p}<0,001)$; coincidiendo con estudios, como Quispe y Quintana, 17,00 $\pm 8,93$ con $\mathrm{p}=0,001$ (20); López y García, $24,88 \pm 9,43$ con $p=0,001$ (21) López et al., 18,83 \pm 9,74 con $\mathrm{p}=0,001$ (12), en Perú; Kramer et al., 3,67 \pm 7,52 con $\mathrm{p}=0,001$ (26); Tello et al., 4,76 con $\mathrm{p}=0,001$ (23), en Brasil; Zambrano et al., 5,81 con $\mathrm{p}=0,001$ en Venezuela (18); Ruano, 17,73 $\pm 6,43$ con $p<0,001$ en Ecuador (28). Los valores de significancia obtenidos a nivel mundial, indican que, la presencia de caries dental tiene un impacto negativo en la calidad de vida en los menores relacionada a la salud bucal, la cual interfiere en distintos ámbitos de su vida; siendo esta problemática asociada a la perdida de sensibilidad y el desinterés de los padres frente al dolor del infante, sumado con la evidente falta de promoción y prevención de programas que no son inclusivos con el grupo etario estudiado; dificultando la detección desde los niveles incipientes de esta patología, para la reducción de sus consecuencias clínicas.

Se concluyó que existe una correlación directa positiva moderada significativa; resultando que, a mayor experiencia y nivel de severidad de caries dental no tratada, lo cual conlleva a un mayor impacto negativo en la calidad de vida a nivel bucal del menor y su entorno. Se encontró que casi la totalidad de los infantes presentaron caries dental con un nivel de severidad muy alto. Las consecuencias clínicas de caries dental no tratada afectó al $83,16 \%$, siendo el componente afección pulpar el de mayor contribución al índice pufa. El impacto fue negativo en la calidad de vida a nivel de salud bucal, donde las dimensiones de mayor puntaje en el impacto en el niño, fueron: limitación funcional y alteraciones psicológicas; así mismo, en el impacto en la familia fue angustia de los padres. El sexo masculino y la edad de 5 años, fueron los más susceptibles y con un nivel de severidad de caries dental no tratada. La presencia de caries dental y sus consecuencias clínicas genera un impacto negativo en la calidad de vida a nivel bucal de los menores y sus familias.

\section{Correspondencia:}

Liz Morales Miranda.

Correo electrónico: lizi2808mm@gmail.com

\section{REFERENCIAS BIBLIOGRÁFICAS}

1. Henostroza G. Caries dental. Principios y procedimientos para el diagnóstico. Madrid: Ripano; 2007.

2. Organización Mundial de la Salud. Salud Bucodental. Centro de Prensa Nota $N^{\circ} 318$. Ginebra: Organización Mundial de la Salud; 2012.

3. Federation Dental International. The Challenge of Oral Disease - A call for global action. The Oral Health Atlas. Geneva: FDI World Dental Federation; 2015.

4. Ministerio de Salud del Perú. Prevalencia nacional de caries dental, fluorosis del esmalte y urgencia de 
tratamiento en escolares de 6 a 8, 10, 12 y 15 años, Perú. Lima: Oficina general de epidemiología y dirección general de salud de las personas; 2005.

5. Villena H. Terapia pulpar. Lima: Universidad Peruana Cayetano Heredia; 2001.

6. Duarte I. Análisis de salud oral y su impacto en la calidad de vida de la población preescolar del municipio de Cascais, Portugal, en 2012. Tesis Doctoral. Sevilla, España: Facultad de odontología de la Universidad de Sevilla; 2015. 178 pp.

7. Otto M. Teeth: The Story of Beauty, Inequality, and the Struggle for Oral Health in America. New York: The New Press; 2017.

8. World Health Organization. WHOQOL- measuring quality of life: The World Health Organization quality of life instruments. Geneva: World Health Organization; 1997.

9. WHOQOL Group. Study protocol for the World Health Organization project to develop a Quality of life assessment instrument (WHOQOL). Qual Life Res. 1993; 2:153-159.

10. Adulyanon S, Sheiham A. Oral impacts on daily performances. In: Slade G. Measuring Oral Health of Quality of Life. Chapell Hill: University of North Carolina, Dental Ecology. 1997; 151-160.

11. Bordoni N, Claravino O, Zambrano O, Villena R, Beltran E, Squassi A. Early Childhood Oral Health Impact Scale (ECOHIS): Translation and validation in spanish language. Acta Odontol Latinoam. 2012; 35(3):270-278.

12. López R, Garcia C, Villena R, Bordoni N. Cross cultural adaptation and validation of the Early Childhood Health Impact Scale (ECOHIS) in peruvian preschoolers. Acta Odontol Latinoam. 2013; 26 (2): 60-67.

13. Pahel B, Rozier R, Slade G. Parental perceptions of children's oral health: the Early Childhood Oral Health Impact Scale. Health Qual Life Outcomes. 2007; 30(5):6.

14. World Health Organization. Oral Health Surveys: Basic Methods. Geneva: World Health Organization; 2013.

15. Monse B, Heinrich R, Benzian H, Holmgren C, Palenstein W. PUFA - An index of clinical consequences of untreated dental caries. Community Dent Oral Epidemiol. 2010; 38: 77-82.

16. Kamran R, Farooq W, Riaz F, Jahangir F. Clinical consequences of untreated dental caries assessed using PUFA index and its covariates in children residing in orphanages of Pakistan. BMC Oral Health. 2017; 17:108.

17. Bansal P, Sujlana A, Pannu P, Kour R. Dental Discomfort Questionnaire: correlated with clinical manifestations of advanced dental caries in young children. J Dent Specialities. 2017; 5(1):3-7.
18. Zambrano O, Fong L, Rivera L, et al. Impacto de la caries de infancia temprana en la calidad de vida del niño zuliano y su familia. Odous Científica. 2015; 16(2):8-17.

19. Alosaimi B, Alturki G, Alnofal S, Alosaimi N, Ansari $\mathrm{S}$. Assessing untreated dental caries among private and public preschool children in Riyadh, a cross-sectional study design. J Dent Oral Health. 2017; 3(10): 1-5.

20. Quispe M, Quintana C. Impacto de la salud oral sobre la calidad de vida en niños preescolares de la institución educativa "José Antonio Encinas“ N. o 1137 en el distrito de Santa Anita, Lima. Revista de Investigación de la Universidad Norbert Wiener. 2017; (6): 61-71.

21. López R, García C. Calidad de vida y problemas bucales en preescolares de la provincia de Huaura, Lima. Rev Estomatol Herediana. 2014; 23(3):139-47.

22. Bagińska J, Rodakowska E, Wilczyńska M, Jamiołkowski J. Index of clinical consequences of untreated dental caries (pufa) in primary dentition of children from north-east Poland. 2013; 58(2):442-447.

23. Tello G, Abant J, Butini L, Murakami C, Bonini G, Bönecker M. Impacto de los principales problemas de salud bucal en la calidad de vida de preescolares. Odontología. 2016; 19(2): 42-52.

24. Patil S, Hathiwala S, Kumar D, Raj S, Khatri S, Singh A. Impact of untreated dental caries on school attendance among the preschool children. International Journal of Current Medical And Pharmaceutical Research. 2016; 8(2):570-572.

25. Mamani V, Padilla T. Riesgo estomatológico e impacto de las afecciones bucales en la calidad de vida de preescolares del ámbito rural en Puno. Odontol Pediatr. 2015; 14 (2): 108-115.

26. Kramer P, Feldens C, Ferreira S, Bervian J, Rodrigues $\mathrm{P}$, Peres M. Exploring the impact of oral diseases and disorders on quality of life of preschool children. Community Dent Oral Epidemiol. 2013; 41: 327-335.

27. Díaz S, Gonzales F, Ramos K, Pérez S, Malo L. Impacto de caries dental sobre la calidad de vida en niños pertenecientes a la primera infancia de instituciones educativas de Cartagena. Tesis Doctoral. Cartagena, Colombia: Facultad de Odontología de Cartagena; 2015. 65 pp.

28. Ruano E. Impacto de los problemas bucodentales en la calidad de vida de niños ecuatorianos de 3 a 5 años de edad. Tesis de Especialidad. Quito, Ecuador: Universidad Central del Ecuador; 2017. 87 pp.
Recibido: 14-07-2018

Aceptado: 12-11-2018 